\title{
RABAH SOUAM
}

\section{Surfaces minimales bordées par quatre droites}

Annales de la faculté des sciences de Toulouse $6^{e}$ série, tome $5, \mathrm{n}^{\mathrm{o}} 1$ (1996), p. 137-153

$<$ http://www.numdam.org/item?id=AFST_1996_6_5_1_137_0>

(C) Université Paul Sabatier, 1996, tous droits réservés.

L'accès aux archives de la revue «Annales de la faculté des sciences de Toulouse » (http://picard.ups-tlse.fr/ annales/) implique l'accord avec les conditions générales d'utilisation (http://www.numdam.org/conditions). Toute utilisation commerciale ou impression systématique est constitutive d'une infraction pénale. Toute copie ou impression de ce fichier doit contenir la présente mention de copyright.

\section{Numdam}

Article numérisé dans le cadre du programme Numérisation de documents anciens mathématiques http://www.numdam.org/ 


\title{
Surfaces minimales bordées par quatre $\operatorname{droites}^{(*)}$
}

\author{
Rabah SoUam ${ }^{(1)}$
}

\begin{abstract}
RÉSUMÉ. - Le but de cet article est de contribuer au problème de la classification des surfaces minimales doublement périodiques plongées dans l'espace euclidien. Nous prouvons que, parmi les surfaces minimales plongées et bordées par quatre droites verticales passant par les sommets d'un parallélogramme horizontal, les surfaces de Scherk et Karcher sont caractérisées nar leur comportement asymtotique.
\end{abstract}

Abstract. - The purpose of this paper is to contribute to the classification problem for doubly periodic embedded minimal surfaces in euclidean space. We prove that, among embedded minimal surfaces bounded by four vertical lines passing through the vertices of a horizontal parallelogram, Scherk and Karcher surfaces are characterized by their asymptotic behaviour.

\section{Introduction}

Soient $P$ un parallélogramme du plan $\left(x_{1}, x_{2}\right)$ de côtés $\left(a, b, a^{\prime}, b^{\prime}\right)$ et $L_{i}, i=1,2,3,4$, les quatre droites verticales passant par les sommets de $P$. On s'intéresse aux surfaces minimales proprement plongées dans $\mathbb{R}^{3}$ et bordées par ces droites. On notera $\widetilde{M}$ une telle surface et on supposera que son intérieur $M$ est situé à l'intérieur du cylindre $P \times \mathbb{R}$.

En faisant subir à $\widetilde{M}$ des réflexions par rapport à chacune de ces droites et en recommençant à chaque fois avec les droites nouvellement obtenues, on génère grâce au principe de réflexion (pour exemple [2]) une surface minimale proprement plongée invariante par les translations des deux vecteurs indépendants $2 a$ et $2 b$. Lorsque l'on quotiente la surface par le groupe engendré par ces deux translations, on obtient une surface minimale proprement plongée dans $\mathbb{T}^{2} \times \mathbb{R}$ où $\mathbb{T}^{2}$ est un tore plat. Des propriétés

(*) Reçu le 3 novembre 1993

(1) Université Paris VII - U.R.A 212 du C.N.R.S., Département de Mathématiques, 2 place Jussieu, F-75251 Paris Cedex 05 (France) 
fondamentales de ces surfaces minimales, dites doublement périodiques, ont été mises en évidence récemment par W. Meeks et H. Rosenberg [5]. Il montrent en particulier qu'une telle surface (minimale et proprement plongée dans $\mathbb{T}^{2} \times \mathbb{R}$ ) de topologie finie est de courbure totale finie (la réciproque est bien connue). Ils montrent de plus que dans ce cas les bouts supérieurs de la surface sont asymptotes à des anneaux plats parallèles et distincts, de même que pour les bouts inférieurs. Par conséquent, si nous voulons que nos surfaces de départ nous fournissent des surfaces de topologie finie dans $\mathbb{T}^{2} \times \mathbb{R}$, nous devons faire une hypothèse sur leur comportement asymptotique.

Les premiers exemples connus de surfaces du type précédent est la famille à un paramètre trouvée par Scherk en 1835 [9]. Dans ce cas $P$ est un losange quelconque et $M$ un graphe sur l'intérieur de $P$ et prend la valeur $+\infty$ sur les côtés $\left(a, a^{\prime}\right)$ et $-\infty$ sur les côtés $\left(b, b^{\prime}\right)$ (figs 1.a et 1.b).

Notons que le problème de Dirichlet pour l'équation des surfaces minimales correspondant admet une solution qui est unique à l'addition d'une constante près : c'est là un cas particulier d'un résultat de $\mathrm{H}$. Jenkins et J. Serrin [1]. Les surfaces obtenues contiennent les droites $L_{i}, i=1,2,3,4$.

Plus récemment, H. Karcher [2] a construit une famille à un paramètre de nouvelles surfaces basées sur des rectangles, ces surfaces ressemblent à deux bandes verticales attachées par une anse horizontale (figs 2.a et 2.b).

Jusqu'à il y a peu de temps les surfaces de Scherk et une famille à trois paramètres qui inclut les surfaces de Karcher ([3] et [5]) étaient les seuls exemples connus. Ceci pose naturellement le problème d'existence d'autres surfaces bordées par les droites verticales issues des sommets d'un parallélogramme horizontal, et constitue un premier pas pour le problème plus général de classification des surfaces minimales doublement périodiques proprement plongées dans $\mathbb{R}^{3}$. Pour les raisons sus-citées nous faisons une hypothèse sur le comportement asymptotique de ces surfaces.

Dans cet article, nous montrerons (théorèmes 2.1 et 3.1 ) que les surfaces de Scherk et celles de Karcher sont en fait caractérisées, parmi ces surfaces, par leur comportement asymptotique. Dans le cas des surfaces de type Scherk, la condition asymptotique est que les bouts supérieurs (resp. inférieurs) sont asymptotes à $\left(a \cup a^{\prime}\right) \times \mathbb{R}^{+}$(resp. à $\left.\left(b \cup b^{\prime}\right) \times \mathbb{R}^{-}\right)$; dans le cas des surfaces de type Karcher, la condition est que les bouts supérieurs (resp. inférieurs) sont asymptotes à $\left(a \cup a^{\prime}\right) \times \mathbb{R}^{+}\left(\right.$resp. à $\left.\left(a \cup a^{\prime}\right) \times \mathbb{R}^{-}\right)$. Ces résultats de rigidité montrent qu'on ne peut pas construire de nouvelles 
surfaces minimales doublement périodiques en ajoutant des anses aux surfaces de Scherk ou de Karcher tout en conservant les droites au bord. Tout récemment, $F$. Wei [11] a réussi à construire une nouvelle famille de surfaces minimales doublement périodiques proprement plongées en "attachant" une anse aux surfaces de Karcher. Utilisant la même idée, $H$. Karcher en a fait de même avec les surfaces de Scherk. Ces surfaces ne contiennent bien entendu pas de droites.

Il est a noter cependant que les surfaces de Karcher existent aussi sur des parallélogrammes non rectangulaires; la technique que nous utilisons, montre dans ce cas, que les surfaces vérifiant nos hypothèses admettent un plan de symétrie horizontal de part et d'autre duquel ces surfaces sont des graphes mais ne permet pas de prouver l'unicité. La question d'existence d'autres exemples, dans ce cas, reste posée.

La démonstration de ces résultats consiste à montrer que la surface est un graphe (dans son intégralité pour les surfaces de type Scherk, par morceaux pour les surfaces de type Karcher); l'unicité découle alors de résultats antérieurs. Pour ce faire, nous utilisons le principe de réflexion d'Alexandrov tel que l'a adapté R. Schoen [10] pour les surfaces minimales. Le problème est rendu délicat par la présence éventuelle de points de branchement pour l'application de Gauss sur les droites du bord et nécessite aussi une bonne compréhension du comportement à l'infini, ce nous détaillons dans la section 1 , le reste de la preuve constitue les sections 2 et 3 .

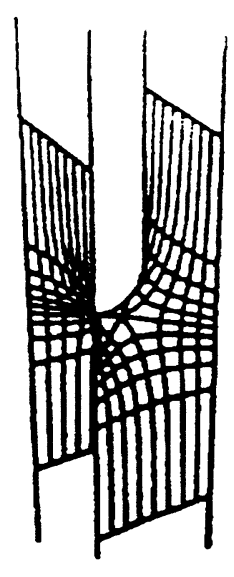

Fig. 1a

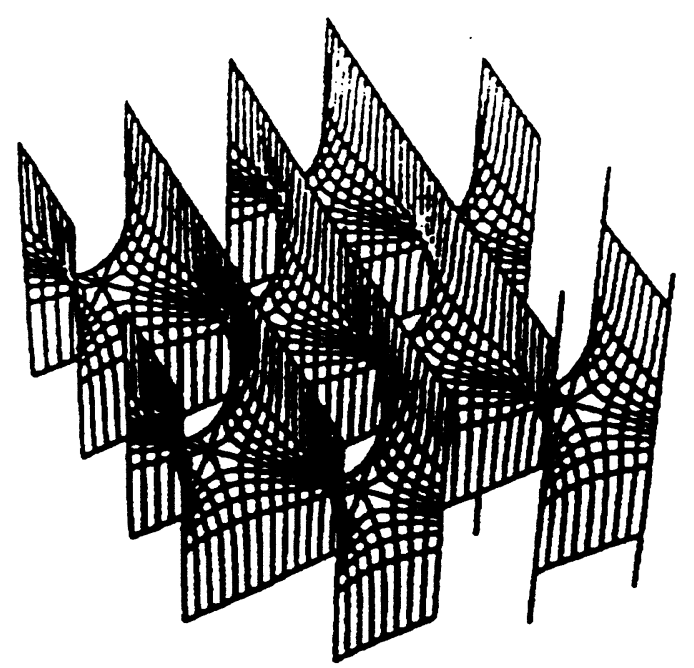

Fig. 1b 


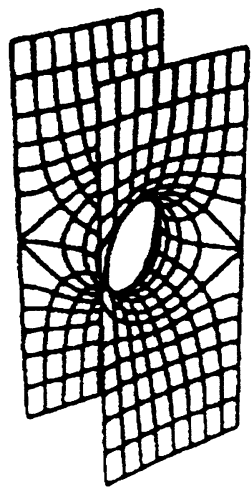

Fig. 2a

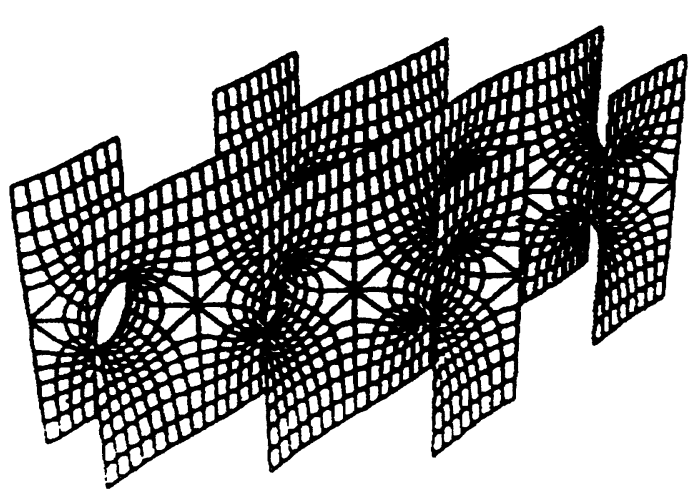

Fig. 2b

\section{Notations - Préliminaires}

On dira qu'une surface à bord $A$ est un graphe au-dessus d'un plan si la projection orthogonale de $A$ sur ce plan est injective. Si de plus le vecteur normal à ce plan n'est tangent à $A$ en aucun point intérieur, on dira que c'est un graphe à pente localement bornée. On dira que $A$ est au-dessus de $B, A \geq B$, si à chaque fois qu'une droite verticale rencontre à la fois $A$ et $B$, tout point de $A$ est au-dessus de tout point de $B$ sur cette verticale. On note pour $t \in \mathbb{R}, A_{t^{+}}=\left\{\left(x_{1}, x_{2}, x_{3}\right) \in A \mid x_{3} \geq t\right\}$ la partie de $A$ située au-dessus du plan $\Pi_{t}$, d'équation $x_{3}=t$. De même, on désigne par $A_{t^{-}}$la partie de $A$ située au-dessous de ce plan et $A_{t^{+}}^{*}$ le symétrique de $A_{t^{+}}$par rapport à $\Pi_{t}$.

Nous aurons à utiliser le principe du maximum pour les surfaces minimales $[10]$ :

- soient $M_{1}$ et $M_{2}$ deux surfaces minimales connexes tangentes en un point intérieur commun $x$ et telles qu'elles soient au voisinage de $x$ d'un même côté l'une de l'autre. $M_{1}$ coïncide alors avec $M_{2}$ au voisinage de $x$ (et $M_{1}$ et $M_{2}$ sont le prolongement analytique l'une de l'autre); 
- supposons que $x$ est un point intérieur à $\partial M_{1}$ et $\partial M_{2}$, que $\partial M_{1}$ et $\partial M_{2}$ sont tangents en $x$ ainsi que $M_{1}$ et $M_{2}$; on a alors la même conclusion que précédemment si $M_{1}$ et $M_{2}$ sont d'un même côté l'une de l'autre près de $x$.

Pour plus de clarté, introduisons la terminologie suivante.

On appellera bande de type Scherk une surface minimale simplement connexe proprement plongée dans $\mathbb{R}^{3}$ dont le bord est constitué de demi-droites parallèles, qu'on pourra supposer dans perte de généralité confondues avec les demi-axes

$$
L_{1}=\left\{x_{1}=x_{2}=0, x_{3} \geq 0\right\} \quad \text { et } \quad L_{2}=\left\{x_{1}=1, x_{2}=0, x_{3} \geq 0\right\},
$$

et d'un arc joignant leurs extrémités. On notera $\widetilde{B}$ une telle bande et $B$ son intérieur. On suppose, de plus, que $\widetilde{B}$ est située d'un même côté (par exemple à droite, dans le sens des $x_{2}$ positifs) du plan $\left(x_{1}, x_{3}\right)$, qu'elle lui est asymptote et enfin qu'elle est contenue dans la région $\left\{0 \leq x_{1} \leq 1\right\}$.

On dira qu'une surface minimale $\widetilde{M}$, bordée par quatre droites verticales passant par les sommets d'un parallélogramme $P$, a le même comportement asymptotique que la surface de Scherk si pour $t$ assez grand $\widetilde{M}_{t^{+}}$est constituée de deux bandes de type Scherk asymptotes à $\left.\left(a \cup a^{\prime}\right) \times \mathbb{R}^{+}\right)$ et $\widetilde{M}_{(-t)}$ - également constitué de deux bandes de type Scherk asymptotes à $\left.\left(b \cup b^{\prime}\right) \times \mathbb{R}^{-}\right)$. Lorsque les bandes supérieures et inférieures sont de type Scherk et sont asymptotes, respectivement à $\left.\left(a \cup a^{\prime}\right) \times \mathbb{R}^{+}\right)$et $\left.\left(a \cup a^{\prime}\right) \times \mathbb{R}^{-}\right)$, nous dirons qu'elle a le même comportement asymptotique que la surface de Karcher.

Nous allons préciser quelques propriétés des bandes de type Scherk qui nous seront utiles par la suite. On a, avec les notations précédentes, le lemme suivant.

\section{LEMME 1.1}

i) Pour $t$ assez grand $\widetilde{B} \cap\left\{x_{3} \geq t\right\}$ est un graphe à pente localement bornée au-dessus du plan $\left(x_{1}, x_{3}\right)$, et $B \cap\left\{x_{3} \geq t\right\}$ un graphe à pente localement bornée au-dessus du plan $\left(x_{1}, x_{2}\right)$.

ii) Pour tout $\varepsilon>0$, il existe $t$ tel que

$$
\widetilde{B} \cap\left\{0 \leq x_{1} \leq \frac{1}{2}-\varepsilon, x_{3} \geq t\right\}
$$

est un graphe à pente localement bornée au-dessus du plan $\left(x_{2}, x_{3}\right)$. 


\section{Rabah Souam}

Preuve. - En applicant le principe de réflexion à $\widetilde{B}$ comme dans l'introduction on obtient une surface minimale $E$ invariante par translation par le vecteur $(2,0,0)$. Soit $G$ le groupe engendré par cette translation. $A=E / G$ est un anneau minimal proprement plongé dans $\mathbb{R}^{3} / G$. W. Meeks et H. Rosenberg [6] ont montré qu'un tel anneau est de courbure totale finie, de plus l'application de Gauss $g$ et la 1-forme holomorphe $\omega$ constituant la représentation de Weierstrass de $E$ sont invariantes par $G$ et donnent donc par passage au quotient une représentation de Weierstrass pour $A$. Comme $A$ est de courbure totale finie, il est conformément équivalent au disque épointé $D^{*}=\{z \in C|0<| z \mid \leq 1\}$ et $(g, \omega)$ s'étendent de façon méromorphe à l'origine [6].

Dans toute la suite de cette preuve, nous supposerons, par commodité, que $\widetilde{B}$ est asymptote à la bande $\left\{0 \leq x_{1} \leq 1, x_{2} \geq 0, x_{3}=0\right\}$. Ceci revient à échanger, dans les énoncés, les rôles de $x_{2}$ et $x_{3}$.

Avec cette convention et avec un choix convenable d'orientation, on a $g(0)=0$ et après reparamétrisation conforme de $A$ on a :

$$
g(z)=z^{p}, \quad \omega(z)=\left(\frac{c_{-q}}{z^{q}}+\frac{c_{-q+1}}{z^{q-1}}+\cdots\right) \mathrm{d} z
$$

avec $p, q \geq 1$.

Ceci montre en particulier que la normale à $\widetilde{B}$ n'est par horizontale donc la projection orthogonale de $\widetilde{B}$ sur le plan $\left(x_{1}, x_{2}\right)$ est un difféomorphisme local, l'hypothèse de propreté entraîne que cette projection est un revêtement donc un difféomorphisme global sur son image qui est simplement connexe; ce qui prouve la première assertion de i).

Passons à la preuve du reste.

Une paramétrisation multivaluée de $E$ est donnée par :

$$
X(z)=\Re \int^{z} \phi
$$

où

$$
\phi=\left(\frac{1-g^{2}}{2}, \frac{i\left(1+g^{2}\right)}{2}, g\right) \omega .
$$

Le vecteur de période étant $v=\Re\left(\int_{S^{1}} \phi\right)$. 
Si $q \neq 1$, on peut écrire :

$$
\left(x_{1}+i x_{2}\right)(z)=\int^{z} \bar{\omega}-\int^{z} g^{2} \omega=\frac{1}{\bar{z}^{q-1}}\left(\frac{\bar{a}}{q-1}+h(z)\right),
$$

avec $a=c_{-q}$ et $h$ une fonction continue s'annulant en zéro. On en déduit que, pour $r$ assez petit, l'argument de $\left(x_{1}+i x_{2}\right)(z)$ subit une variation de $2 \pi(q-1)$ lorsque $z$ décrit une fois le cercle $|z|=r$. Or $B$ est asymptote à la bande $\left\{0 \leq x_{1} \leq 1, x_{2} \geq 0, x_{3}=0\right\}$ et ainsi l'argument de $\left(x_{1}+i x_{2}\right)(z)$ ne peut subir une variation supérieure à $\pi$ lorsque $z$ décrit une fois le cercle $|z|=r$. Ainsi a-t-on $q=1$.

Vu l'orientation choisie sur le graphe $B$ (normale dirigée vers le bas), le vecteur de période est $v=(-2,0,0)$ (lorsque $z$ décrit le cercle $|z|=r$ dans le sens positif, la coordonnée $x_{1}(z)$ décroît). Ceci entraîne que $a=2 i / \pi$.

La paramétrisation de $E$ est ainsi de la forme :

$$
\begin{aligned}
& x_{1}(z)=-\frac{1}{\pi} \arg (z)+\alpha_{1}(z) \\
& x_{2}(z)=-\frac{1}{\pi} \ln |z|+\alpha_{2}(z) \\
& x_{3}(z)=-\frac{2}{p \pi} \Re\left(i z^{p}+\operatorname{ord}\left(z^{p}\right)\right.
\end{aligned}
$$

$\alpha_{1}, \alpha_{2}$ étant deux fonctions continues en 0 . Comme $x_{3}$ change de signe deux fois dans $A$ on a $p=1$.

Les points de $A$ dont le plan tangent contient le vecteur $(0,1,0)$ correspondent aux valeurs du paramètre $z$ telles que $\Im g(z)=0$, c'est-à-dire à deux courbes connexes, il n'y a donc sur $\widetilde{B}$ que les points $L_{1}$ et $L_{2}$ dont le plan tangent contient le vecteur $(0,1,0)$, il s'ensuit que $B$ est un graphe au-dessus du plan $\left(x_{1}, x_{3}\right)$. En effet, dans le cas contraire il existerait deux points $p$ et $q$ sur $B$ situés sur une même droite horizontale. $B$ étant un graphe au-dessus du plan $\left(x_{1}, x_{2}\right)$, il existerait par continuité sur l'arc de $B$ qui se projette sur le segment $[p, q]$, un point où le vecteur $(0,1,0)$ est tangent à $B$ ce qui est exclu. Ceci prouve la seconce assertion de i).

Pour prouver ii) cherchons, de même, les points de $A$ dont le plan tangent contient le vecteur $(1,0,0)$. Cette condition s'exprime par $\Re g(z)=0$, c'està-dire par $z \in i \mathbb{R}$. Ces points constituent donc deux courbes $\gamma_{1}$ et $\gamma_{2}$ sur $A$. Par ailleurs, $L_{1}$ et $L_{2}$ correspondent respectivement à $z \in \mathbb{R}^{+}$et $\mathbb{R}^{-} \cdot \gamma_{1}$ et $\gamma_{2}$ n'intersectent donc ni $L_{1}$, ni $L_{2}$; ceci joint au fait que la condition que le plan tangent contient le vecteur $(1,0,0)$ est conservée par symétrie par 
rapport à $L_{1}$ entraîne que l'une des courbes, disons $\gamma_{1}$, est dans $B$ et $\gamma_{2}$ est sa symétrique par rapport à $L_{1}$. Cette courbe $\gamma_{1}$ correspond à $z \in i \mathbb{R}^{-}$.

On a :

$$
0=x_{1}(r, 0)=\alpha_{1}(r, 0)
$$

et comme $\alpha_{1}$ est continue en 0 :

$$
\lim _{r \rightarrow 0} x_{1}\left(r,-\frac{\pi}{2}\right)=\frac{1}{2}+\lim _{r+0} \alpha_{1}\left(r,-\frac{\pi}{2}\right)=+\frac{1}{2} .
$$

Ceci entraîne que pour tout $\varepsilon>0$, il existe $t$ tel que le vecteur $(1,0,0)$ ne soit tangent à $\widetilde{B} \cap\left\{0<x_{1}<1 / 2-\varepsilon, x_{2}>t\right\}$ en aucun point, ce qui termine la preuve de ii) par le même argument que précédemment.

\section{Surfaces de Scherk}

THÉORÈME 2.1. - Soit $\widetilde{M}$ une surface minimale connexe proprement plongée dans $\mathbb{R}^{3}$ bordée par quatre droites verticales issues des sommets d'un parallélogramme $P$ du plan $\left(x_{1}, x_{2}\right)$ et dont l'intérieur $M$ se trouve à l'intérieur du cylindre $P \times \mathbb{R}$. Supposons que $\widetilde{M}$ a le même comportement asymtotique que la surface de Scherk alors $P$ est un losange, l'intérieur $M$ de $\widetilde{M}$ est un graphe qui se projette sur $P$ et ainsi $\widetilde{M}$ est la surface de Scherk.

Preuve. - Le fait que $P$ soit un losange sera une conséquence du fait que $M$ est un graphe se projettant sur $P$, car c'est par le résultat de Jenkins-Serrin une condition nécessaire (et suffisante) pour que le problème de Dirichlet correspondant admette une solution. On peut aussi le démontrer directement comme il ressort de [5], nous en esquissons la preuve. Pour $t$ assez grand $M(t)=M \cap(P \times[-t, t])$ est une surface compacte dont le bord est constitué de quatre segments verticaux, de deux courbes disjointes sur $P \times\{t\}$ proches des segments $\left(a, a^{\prime}\right)$ (par exemple) et de deux courbes similaires sur $P \times\{-t\}$ et proches des segments $\left(b, b^{\prime}\right)$. La surface $M(t)$ étant minimale, ses fonctions coordonnées sont harmoniques et donc le flux du champ de vecteurs projection sur $M(t)$ du champ constant $(0,, 0,1)$ à travers $\partial M(t)$ est nul. À l'infini ce flux vaut $|a|+\left|a^{\prime}\right|-|b|-\left|b^{\prime}\right|$ d'où $|a|=|b|$.

Nous allons montrer maintenant que $M$ est un graphe sur une partie de $P$; il est cependant facile de voir que la surface se projette sur $P$ tout entier vu l'hypothèse sur son comportement asymptotique et sur son bord. 
Ceci montrera que $\widetilde{M}$ est la surface de Scherk en utilisant le résultat de Jenkins-Serrin.

Pour ce faire considérons l'ensemble $I$ des $t \in \mathbb{R}$ tels que $M_{t^{+}}$est un graphe à pente localement bornée au-dessus de $\Pi_{t}$ et $M_{t^{+}}^{*} \geq M_{t^{-}}$. Remarquons que si $t \in I$ alors $[t,+\infty) \subset I$, donc $I$ est un intervalle. Nous devons montrer que $I=\mathbb{R}$.

On a $I \neq \emptyset$ : on a vu (lemme $1.1 \mathrm{i})$ ) que pour $t$ assez grand $M_{t^{+}}$est un graphe à pente localement bornée au-dessus de $\Pi_{t}$. Par ailleurs, les deux composantes de $M_{t^{+}}$ainsi que leurs plans tangents le long des droites au bord tendent uniformément vers deux faces opposées du cylindre $P \times \mathbb{R}$ et $M_{(-t)}$ - a le même comportement avec les deux autres faces de ce cylindre. Il s'ensuit que $M_{t^{+}}$est disjoint de $M_{(-t)}$ - pour $t$ assez grand. Fixons un tel réel $t_{1}$, notons que l'on peut aussi assurer que, pour tout $t \geq t_{1}$ :

$$
M_{t^{+}}^{*} \geq M_{\left(-t_{1}\right)^{-}} .
$$

Le long des points de $\partial M\left(t_{1}\right)$ qui se trouvent sur les droites verticales, le plan tangent à la surface est strictement entre les faces de $P \times \mathbb{R}$ (par le principe du maximum à bord; sinon $\widetilde{M}$ serait une partie du bord du cylindre $P \times \mathbb{R}$ ) et comme une nouvelle fois le plan tangent à $M_{t_{1}^{+}}$le long des droites au bord tend uniformément vers une face de $P \times \mathbb{R}$, on peut, pour $t \geq t_{1}$ et assez grand, trouver un voisinage $U$ des quatre segments de $\partial M\left(t_{1}\right)$ dans $M\left(t_{1}\right)$ tel que $M_{t^{+}}^{*} \geq U$. La partie $M\left(t_{1}\right) \backslash U$ est maintenant compacte et disjointe des faces de $P \times \mathbb{R}$ par le principe du maximum intérieur; elle sera donc disjointe de $M_{t^{+}}^{*}$ pour $t$ assez grand. Par ailleurs, $M_{t_{1}^{+}}$étant un graphe, pour $t>t_{1}$ on a

$$
M_{t^{+}}^{*} \geq M \cap\left(P \times\left[t_{1}, t\right]\right) .
$$

Il résulte de ce qui précède que, pour $t$ assez grand, $M_{t^{+}}^{*} \geq M_{t^{-}}$.

Considérons maintenant $t_{0}=\inf I$. Supposons $t_{0}$ fini, nous allons aboutir à une contradiction ce qui montrera que $M$ est un graphe.

Nous commençons par montrer que $t_{0} \in I$. La preuve est la même que dans [10], nous la rappelons brièvement : on a $M_{t_{0}^{+}}^{*} \geq M_{t_{0}^{-}}$car autrement il y aurait deux points $\left(x_{1}, x_{2}, x_{3}\right) \in M_{t_{0}^{+}}$et $\left(x_{1}, x_{2}, y_{3}\right) \in M_{t_{0}^{-}}$tels que $2 t_{0}-x_{3}<y_{3}$. Il en découle que $x_{3}>t_{0}$, mais ceci entraîne que l'on n'a pas $M_{s^{+}}^{*} \geq M_{s^{-}}$pour $s>t_{0}$ et suffisamment proche de $t_{0}$, ce qui constitue une contradiction. 
D'autre part, si deux points $\left(x_{1}, x_{2}, x_{3}\right)$ et $\left(x_{1}, x_{2}, y_{3}\right)$ appartiennent tous deux à $M_{t_{0}^{+}}$avec $y_{3}<x_{3}$ alors nécessairement $y_{3}=t_{0}$. Le plan tangent à $M$ au point $\left(x_{1}, x_{2}, x_{3}\right)$ n'étant pas vertical, il existe dans $M$ un voisinage de ce point qui est un graphe se projettant sur un voisinage de $\left(x_{1}, x_{2}, t_{0}\right)$ dans le plan $\Pi_{t_{0}}$. Comme $M_{s}$ est, de plus, un graphe pour tout $s>t$, on en déduit facilement qu'un voisinage de $\left(x_{1}, x_{2}, t_{0}\right)$ dans $M$ reste au-dessous du plan $\Pi_{t_{0}}$, ce qui contredit le principe du maximum intérieur, aussi $M_{t_{0}^{+}}$ est-il un graphe au-dessus de $\Pi_{t_{0}}$.

Soit $p=\left(x_{1}, x_{2}, t_{0}\right)$ un point de $M$; le plan tangent $T_{p} M$ n'est pas vertical, sinon, par le principe du maximum à bord, ou aurait $M_{t_{0}^{+}}^{*}=M_{t_{0}^{-}}$ et $\Pi_{t_{0}}$ serait ainsi un plan de symétrie pour $\widetilde{M}$, ce qui est en contradiction avec son comportement asymptotique. Ainsi $M$ reste un graphe au voisinage de tout point de $M \cap \Pi_{t_{0}}$, mais ceci ne nous suffit pas et nous devons tenir compte des sommets de $P \times\left\{t_{0}\right\}$.

Considérons donc un des sommets $p$ du parallélogramme $P \times\left\{t_{0}\right\}$. Nous allons montrer que $M$ reste un graphe au voisinage de $p$. Notons $M_{1}$ la surface réunion de $\widetilde{M}$ et de sa symétrique par rapport à la droite du bord qui contient $p$. Notons, de même, $M_{2}$ la symétrique de $M_{1}$ par rapport au plan $\Pi_{t_{0}} . M_{1}$ et $M_{2}$ sont deux surfaces minimales (régulières) tangentes en $p$ et qui s'intersectent au voisinage de $p$ exactement en deux courbes : $M_{1} \cap \Pi_{t_{0}}$ et la droite verticale $x_{1}=x_{1}(p), x_{2}=x_{2}(p)$. Après rotation et translation de $\mathbb{R}^{3}$ ramenant $p$ à l'origine et $T_{p} M$ sur le plan $\left(x_{1}, x_{2}\right)$, on peut représenter localement $M_{i}, i=1,2$, par $x_{3}=u_{1}\left(x_{1}, x_{2}\right), i=1,2$, où les fonctions $u_{i}$ sont solutions de l'équation des surfaces minimales et s'annulent ainsi que leurs premières dérivées à l'origine. Si $p$ était un point de branchement pour l'application de Gauss de $M_{1}$ (et par conséquent aussi pour $M_{2}$ ) leurs dérivées secondes seraient aussi nulles (en un point de branchement de l'application de Gauss sur une surface minimale, la seconde forme fondamentale est, en effet, identiquement nulle). Ainsi $p$ serait un point de contact d'ordre au moins deux des deux surfaces minimales $M_{1}$ et $M_{2}$. Or ceci entraîne [7, p. 394] qu'elles s'intersectent en au moins trois arcs analytiques passant par $p$, qui localement divisent tout voisinage de $p$ en au moins six secteurs dans lesquels $M_{2}$ est alternativement d'un côté et de l'autre de $M_{1}$, ce qui constitue une contradiction. Ainsi $p$ n'est pas un point de branchement pour l'application de Gauss de $M_{1}$. On en déduit qu'au voisinage de $p$ le plan tangent à $\widetilde{M}$ n'est vertical que le long de la droite du bord (l'application de Gauss est en effet un difféomorphisme conforme au voisinage de $p$ ). Il en résulte par le même argument que dans la preuve 
du lemme 1.1 (deuxième assertion de i), en utilisant cette fois-ci le fait que $M$ est localement un graphe au-dessus de $T_{p} M$, qui est vertical) qu'au voisinage de $p, M$ reste un graphe à pente localement bornée au-dessus du plan $\left(x_{1}, x_{2}\right)$.

Des deux points précédents, il découle qu'il existe un voisinage de $U$ de $\Pi_{t_{0}}$ tel que $M \cap U$ est un graphe à pente localement bornée au-dessus du plan $\left(x_{1}, x_{2}\right)$. Pour contredire la finitude de $t_{0}$ il nous reste à vérifier que $M_{s^{+}}^{*} \geq M_{s^{-}}$pour $s<t_{0}$ et suffisamment proche de $t_{0}$. Comme $M \cap U$ est un graphe, on a $M_{s^{+}}^{*} \cap U \geq M_{s^{-}}$pour un voisinage $V$ de $\Pi_{t_{0}}, V \subset U$ de la forme $\left\{t_{0}-\alpha \leq x_{3} \leq t_{0}+\alpha\right\}$ et pour $s$ proche de $t_{0}$. Considérons la partie $D=M_{s^{+}} \backslash V$ qui ne dépend pas de $s$ proche de $t_{0}$ et coïncide en fait avec $M_{\left(t_{0}+\alpha\right)^{+}}$. Notons $\rho_{s}$ la symétrie par rapport au plan $\Pi_{s}$. On a

$$
M_{s^{+}}^{*}=\left(M_{s^{+}}^{*} \cap V\right) \cup \rho_{s}(D) .
$$

Nous venons de voir que pour $s<t_{0}$ et suffisamment proche de $t_{0}$ :

$$
M_{s^{+}}^{*} \cap V \geq M_{s^{-}}
$$

il nous reste à montrer que $\rho_{s}(D) \geq M_{s^{-}}$, ce qui sera a fortiori vrai si nous montrons que $\rho_{s}(D) \geq M_{t_{0}^{-}}$(car pour $s<t_{0}, M_{s^{-}} \subset M_{t_{0}^{-}}$). Notons que, pour $s<t_{0}$

$$
\rho_{s}(D)=\rho_{t_{0}}(D)+\left(0,0, s-t_{0}\right)
$$

nous avons donc à prouver que pour $\varepsilon>0$ suffisamment petit $\rho_{t_{0}}(D)+$ $(0,0,-\varepsilon) \geq M_{t_{0}^{-}}$.

Comme $t_{0} \in I, \rho_{t_{0}}(D) \geq M_{t_{0}^{-}}$; de plus, ces deux ensembles sont disjoints en vertu du principe du maximum intérieur. Pour $t$ assez grand $M_{\left(t_{0}-t\right)^{-}}$ et $\rho_{t_{0}}(D)$ sont disjoints et proches ainsi que leurs plans tangents de faces distinctes du cylindre $P \times \mathbb{R}$, ils restent donc disjoints par une petite translation verticale de $\rho_{t_{0}}(D)$ : pour $\varepsilon$ assez petit $\rho_{t_{0}}(D)+(0,0,-\varepsilon)$ et $M_{\left(t_{0}-t\right)}$ - restent disjoints. Considérons maintenant l'ensemble

$$
M^{\prime}=M \cap\left(P \times\left[t_{0}-t, t_{0}\right]\right)
$$

où $t$ est fixé et assez grand. $M^{\prime}$ est relativement compacte et disjoint de $\rho_{t_{0}}(D)$. Le principe du maximum à bord implique que, en chaque point des segments du bord de $M^{\prime}$, le plan tangent à $M^{\prime}$ est distinct du plan tangent au même point à $\rho_{t_{0}}(D)$. Ceci joint au comportement asymptotique 
de $\rho_{t_{0}}(D)$, entraîne là aussi que, pour $\varepsilon>0$ assez petit, $\rho_{t_{0}}(D)+(0,0,-\varepsilon)$ reste disjoint de $M^{\prime}$. On vient de voir donc que, pour tout $\varepsilon>0$ assez petit, $\rho_{t_{0}}(D)+(0,0,-\varepsilon)$ reste disjoint de $M_{t_{0}^{-}}\left(\operatorname{car} M_{t_{0}^{-}}=M^{\prime} \cup M_{\left(t_{0}-t\right)^{-}}\right)$et donc que $\rho_{t_{0}}(D)+(0,0,-\varepsilon) \geq M_{t_{0}^{-}}\left(\right.$dans le cas contraire, $\rho_{t_{0}}(D)+\left(0,0,-\varepsilon^{\prime}\right)$ et $M_{t_{0}}$ ne seraient pas disjoints pour un certain $\varepsilon^{\prime}<\varepsilon$ ). En conclusion, pour $s<t_{0}$ et assez proche de $t_{0}$, on a $s \in I$, ce qui contredit le fait de $t_{0}=\inf I$ et ainsi $t_{0}=-\infty$.

\section{Remarques}

i) Un cas particulier des résultats de Jenkins-Serrin [1] est qu'étant donné un polygone convexe $P$ ayant un nombre pair de côtés et de même longueur, il existe à l'addition d'une constante près un unique graphe minimal audessus de $P$ et qui prend alternativement les valeurs $+\infty,-\infty$ sur les côtés de $P$. La surface minimale ainsi obtenue est bordée par les droites verticales passant par les sommets de $P$. La preuve du théorème 2.1 se généralise sans changement à cette situation et permet de caractériser ces "surfaces de Scherk généralisées" par leur comportement asymptotique parmi les surfaces minimales proprement plongées bordées par les droites verticales issues des sommets de $P$ et contenues dans $P \times \mathbb{R}$.

ii) Signalons que R. Langevin, G. Levitt et H. Rosenberg [4] ont caractérisé la surface de Scherk par le fait que son intérieur soit un graphe au-dessus de $P$ bordé par les droites $L_{i}, i=1,2,3,4$.

\section{Surfaces de Karcher}

Dans le cas des surfaces de Karcher les parallélogrammes $P$ sont des rectangles avec $|a|>|b|$ et on a pour chacun un graphe minimal au-dessus du sous-domaine de $P$ bordé par les côtés $\left(a, a^{\prime}\right)$ et deux courbes convexes $C_{1}$ et $C_{2}$ (fig. 3). La fonction est nulle sur $C_{1}, C_{2}$ et vaut $+\infty$ sur $\left(a, a^{\prime}\right)$, de plus le graphe est vertical le long de $C_{1}$ et $C_{2}$. Par le principe de réflexion [2], ce graphe se prolonge par symétrie par rapport au plan de $P$. H. Karcher prouve l'existence de ces surfaces à partir des surfaces conjuguées qui sont des graphes de Jenkins-Serrin par morceaux (une autre construction de ces mêmes surfaces est due à W. Meeks et H. Rosenberg [6] et prouve aussi l'existence des surfaces de Karcher sur des parallélogrammes non rectangulaires. Elle consiste à obtenir la surface de Karcher comme limite d'une suite d'anneaux minimaux bordés par des courbes convenablement 
choisies sur les bandes $(a) \times \mathbb{R}$ et $\left(a^{\prime}\right) \times \mathbb{R}$. Ce procédé aboutit dans le cas où il existe une caténoïde minimisante dont le bord se trouve sur les bandes précédentes).

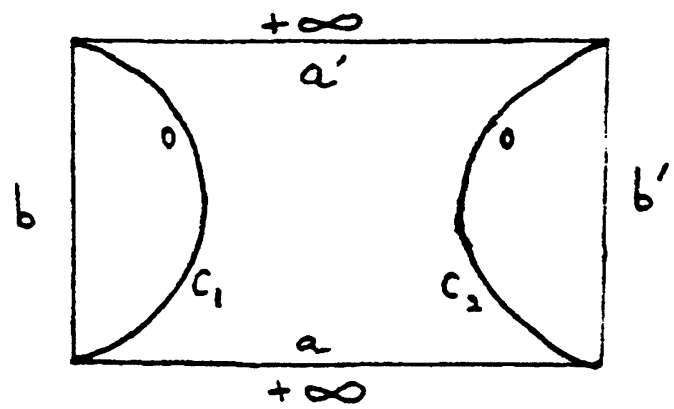

Fig. 3

Nous passons maintenant à la caractérisation de ces surfaces.

THÉORÈME 3.1.- Soit $\widetilde{M}$ une surface minimale connexe proprement plongée dans $\mathbb{R}^{3}$ bordée par quatre droites verticales issues des sommets d'un rectangle $P$ du plan $\left(x_{1}, x_{2}\right)$ et dont l'intérieur est situé à l'intérieur du cylindre $P \times \mathbb{R}$. Si $\widetilde{M}$ a le même comportement asymptotique que les surfaces de Karcher aors $\widetilde{M}$ est une surface de Karcher.

Preuve. - On procède de la même façon que précédemment pour montrer que $\widetilde{M}$ admet un plan symétrie horizontal, de part et d'autre duquel son intérieur $M$ est un graphe.

En reprenant les mêmes notations, le fait que l'ensemble $I$ n'est pas vide se démontre de la même manière. Le seul point à vérifier est le fait suivant que nous énonçons sous forme de lemme.

Lemme 3.2.- Soient $B_{1}$ et $B_{2}$ deux bandes de type Scherk s'appuyant sur deux mêmes demi-droites contenues dans le plan $\left(x_{1}, x_{2}\right)$, alors on peut translater horizontalement $B_{1}$ de sorte qu'elle soit située en-dessous de $B_{2}$.

Preuve du lemme 3.2.- Notons que, d'une part $B_{1}$ et $B_{2}$ sont des graphes asymptotes au plan $\left(x_{1}, x_{2}\right)$ et situés, par exemple, au-dessus de celui-ci et d'autre part par le principe du maximum à bord, le plan tangent le long des demi-droites n'est jamais confondu avec le plan $\left(x_{1}, x_{2}\right)$ mais tend uniformément vers celui-ci. On peut donc translater $B_{1}$ de sorte que 
son bord soit situé en-dessous de celui de $B_{2}$. Nous allons voir que, après cette translation, $B_{2} \geq B_{1}$. Vu le comportement asymptotique de $B_{1}$ et $B_{2}$, on a pour $t>0$ assez grand $B_{2}+(0,0, t) \geq B_{1}$, désignons par $\eta \geq 0$ l'infimum de tels $t$. Nous devons montrer que $\eta=0$. Supposons $\eta>0$ et notons que dans ce cas $B_{1}+(0,0, \eta)$ et $B_{2}$ ne se rencontrent pas grâce au principe du maximum intérieur (rappelons que les bords sont disjoints). Le comportement asymptotique de $B_{1}$ et $B_{2}$ implique que, pour $R>0$ assez grand, $B_{1}(R):=B_{1} \cap\left\{x_{2}>R\right\}$ est contenu dans $\left\{x_{3} \leq t_{0} / 2\right\}$, ainsi $B_{1}(R) \leq B_{2}+(0,0, \eta / 2)$. L'ensemble $B^{\prime}:=B_{1} \backslash B_{1}(R)$ est compact et est situé strictement en-dessous de $B_{2}+(0,0, \eta)$, ceci joint à l'hypothèse de propreté sur $B_{2}+(0,0, \eta)$ montre que, pour $\varepsilon>0$ assez petit, $B_{2}+(0,0, \eta-\varepsilon) \geq B^{\prime}$. Ainsi pour $0<\varepsilon<\eta / 2$ et assez petit $B_{2}+(0,0, \eta-\varepsilon) \geq B_{1}$, ce qui est contradictoire et donc $\eta=0$.

Poursuivons la preuve du théorème en notant que $t_{0}=\inf I$ doit être fini car $M$ n'est évidemment pas un graphe. L'argument utilisé dans la preuve du théorème 3.1 montre alors qu'un des sommets au moins de $P \times\left\{t_{0}\right\}$ doit être un point de branchement pour l'application de Gauss de $\widetilde{M}$ et donc le plan $\Pi_{t_{0}}$ doit être un plan de symétrie pour $\widetilde{M}$ de part et d'autre duquel $M$ est un graphe (notons que ceci a pour conséquence que les quatre sommets de $P \times\left\{t_{0}\right\}$ sont des points de branchement de l'application de Gauss).

Par le même procédé, nous allons montrer que $\widetilde{M}$ est un bigraphe, invariant par symétrie par rapport au plan de symétrie vertical du cylindre $P \times \mathbb{R}$ orthogonal à $\left(a, a^{\prime}\right) \times \mathbb{R}$.

Pour garder les mêmes notations, faisons une rotation de l'espace et une homothétie pour que notre rectangle devienne le rectangle vertical

$$
P=\left\{0 \leq x_{1} \leq \ell, x_{2}=0,0 \leq x_{3} \leq 1\right\}
$$

et que $\widetilde{M}$ soit asymptote aux deux faces verticales de $P \times \mathbb{R}$.

L'intervalle $I$ est d'intérieur non vide grâce d'une part au lemme 1.1 i) et d'autre part au fait que le long des droites au bord le plan tangent à $\widetilde{M}$ n'est jamais vertical par le principe du maximum à bord et donc sur toute partie compacte de $\widetilde{M}$, il y a un voisinage des segments au bord qui est un graphe à pente localement bornée au-dessus d'une partie du plan $\left(x_{1}, x_{2}\right)$.

Nous allons voir que $1 / 2 \in I$. Ceci revient à montrer que

$$
\begin{gathered}
t_{0}=\inf I \leq \frac{1}{2} . \\
-150-
\end{gathered}
$$


De la même manière que la preuve du théorème 2.1 on a $\widetilde{M}_{t_{0}^{+}}^{*} \geq \widetilde{M}_{t_{0}^{-}}$. Supposons $t_{0}>1 / 2$, alors le plan $\Pi_{t_{0}}$ n'étant pas un plan de symétrie pour $\widetilde{M}$, on en déduit, par le principe du maximum à bord, que le plan tangent à $\widetilde{M}$ aux points de $\widetilde{M} \cap \Pi_{t_{0}}$ n'est pas vertical. Par conséquent chacun de ces points possède un voisinage dans $\widetilde{M}$ qui est un graphe à pente localement bornée au-dessus du plan $\left(x_{1}, x_{2}\right)$. Ceci, avec le lemme 1.1 ii), montre qu'il existe $\varepsilon>0$ tel que $\widetilde{M}_{\left(t_{0}-\varepsilon\right)^{+}}$est un graphe à pente localement bornée au-dessus du plan $\left(x_{1}, x_{2}\right)$.

Ainsi pour $t_{0}-\varepsilon / 2<s \leq t_{0}$, on a :

$$
\widetilde{M}_{s^{+}}^{*} \cap\left\{t_{0}-\varepsilon<x_{3} \leq t_{0}\right\} \geq \widetilde{M}_{s^{-}} .
$$

Nous devons montrer que

$$
\widetilde{M}_{s^{+}}^{*} \backslash\left\{t_{0}-\varepsilon<x_{3} \leq t_{0}\right\}\left(=\widetilde{M}_{t_{0}^{+}}^{*}+\left(0,0,2\left(s-t_{0}\right)\right) \cap\left\{x_{3} \leq t_{0}-\varepsilon\right\}\right)
$$

ne rencontre pas $\widetilde{M}_{\left(t_{0}-\varepsilon\right)^{-}}$pour $s$ proche de $t_{0}$. Nous savons que $\widetilde{M}_{t_{0}^{+}}^{*}$ ne rencontre pas $\widetilde{M}_{\left(t_{0}-\varepsilon\right)}$ - et d'autre part que $\widetilde{M}_{\left(t_{0}-\varepsilon_{0}\right)}+$ est un graphe, donc, pour $s$ assez proche de $t_{0}$, le bord de

$$
\widetilde{M}_{t_{0}^{+}}^{*}+\left(0,0,2\left(s-t_{0}\right)\right) \cap\left\{x_{3} \leq t_{0}-\varepsilon\right\}
$$

ne rencontre pas $\widetilde{M}_{\left(t_{0}-\varepsilon\right)}$ - et inversement. Le principe du maximum montre alors qu'ils sont disjoints (translater $\widetilde{M}_{t_{0}^{+}}^{*}+\left(0,0,2\left(s-t_{0}\right)\right) \cap\left\{x_{3} \leq t_{0}-\varepsilon\right\}$ horizontalement et assez loin dans le sens des $x_{1}$ négatifs puis la ramener à sa position initiale. Par le comportement asymptotique des deux surfaces, si elles ne sont pas disjointes, il devrait y avoir un premier point (intérieur) de contact; elles seraient ainsi identiques jusqu'au bord!).

On aboutit donc à une contradiction avec le fait que $t_{0}=\inf I$. Ainsi $t_{0} \leq 1 / 2$ et $\widetilde{M}_{(1 / 2)}+$ est un graphe au-dessus du plan $\left(x_{1}, x_{2}\right)$ et $\widetilde{M}_{(1 / 2)}+\geq$ $\widetilde{M}_{(1 / 2)^{-}}$.

Il suffit maintenant de refaire le même raisonnement par le bas. On a donc également $\widetilde{M}_{(1 / 2)^{-}}^{*} \leq \widetilde{M}_{(1 / 2)^{+}}$, c'est-à-dire $\widetilde{M}_{(1 / 2)^{+}}^{*} \leq \widetilde{M}_{(1 / 2)^{-}}$. Le plan de symétrie vertical du cylindre $P \times \mathbb{R}$ orthogonal aux bouts de $\widetilde{M}$ est donc un plan de symétrie pour $\widetilde{M}$.

La même méthode de réflexion montre (c'est même plus simple dans ce cas) que le deuxième plan de symétrie vertical de cylindre $P \times \mathbb{R}$ est aussi un plan de symétrie pour $\widetilde{M}$, de part et d'autre duquel celle-ci est un graphe. 
L'une quelconque des quatre parties de la surface comprise entre les trois plans de symétrie est un graphe au-dessus de chacun de ces plans, on en déduit facilement que son bord est connexe constitué d'une demi-droite et des trois arcs sur les plans de symétrie et qui sont des graphes monotones. Il en résulte que $\widetilde{M}$ a la même topologie que les surfaces de Karcher.

On prolonge la surface par symétries répétées par rapport aux droites du bord. La surface $N$ dans $\mathbb{T}^{2} \times \mathbb{R}$ obtenue en quotientant par le groupe engendré par les translations des vecteurs $2 a$ et $2 b$ est donc topologiquement le tore moins quatre points.

Meeks et Rosenberg [5] ont montré que pour les surfaces minimales proprement plongées dans $\mathbb{T}^{2} \times \mathbb{R}$, la courbure totale $C(M)$ et la caractéristique d'Euler sont liées par :

$$
C(N)=2 \pi \chi(N)
$$

Par ailleurs $C(N)=4 \pi \operatorname{deg}(\bar{g}) ; \bar{g}$ étant l'extension de l'application de Gauss à la compactifiée de $N$. On en déduit que $\operatorname{deg}(\bar{g})=2$ et la formule de Riemann-Hurwitz implique que $\bar{g}$ possède quatre points de branchement. Ainsi l'application de Gauss de $\widetilde{M}$ n'admet pas de points de branchement autres que les quatre sommets de son intersection avec son plan de symétrie horizontal; ce sont donc les seuls points de courbure nulle de $\widetilde{M}$. En particulier, les courbes d'intersection de $\widetilde{M}$ avec les plans de symétrie n'admettent pas de points d'inflexion.

La partie de $P$ bordée par $a, a^{\prime}$ et les deux courbes convexes d'intersection de la surface avec le plan de symétrie horizontal est certainement contenue dans la projection de la surface. D'autre part si elle se projettait à l'extérieur de ce domaine on aurait une contradiction avec le fait que, en-dehors des points du plan de symétrie horizontal, le plan tangent à $M$ n'est jamais vertical. On retrouve bien les surfaces de Karcher.

\section{Remerciements}

Cet article reprend une partie de la thèse de l'auteur (Université Paris VII, 1992). Je tiens à remercier le professeur H. Rosenberg pour l'aide et les conseils qu'il m'a apportés. Les figures utilisées sont tirées de [8]. 


\section{Bibliographie}

[1] JENKINS (H.) et SERRIN (J.) . - Variational problems of minimal surface type II, Arch. Rat. Mech. Analysis 21 (1966), pp. 321-342.

[2] KARCHER (H.) .- Embedded minimal surfaces derived from Scherk's examples, Manus. Math. 62 (1988), pp. 83-114.

[3] KARCher (H.) .- Construction of minimal surfaces, Surveys in Geometry, University of Tokyo (1989), pp. 1-96.

[4] Langevin (R.), Levitt (G.) et Rosenberg (H.) .- Complete minimal surfaces with long line boundaries, Duke Math. Journal 55 (1987), pp. 77-84.

[5] Meeks III (W.) et Rosenberg (H.) . - The global theory of doubly periodic minimal surfaces, Invent. Math. 97 (1989), pp. 351-379.

[6] Meeks III (W.) et Rosenberg (H.) .- The geometry of periodic minimal surfaces, Comment. Math. Helv. 68 (1993), pp. 538-578.

[7] Nitsche (J. C. C.) .- Lectures on minimal surfaces, Vol. 1, Cambridge University Press.

[8] Rosenberg (H.) .- Some recent developements in the theory on properly embedded minimal surfaces in $\mathbb{R}^{3}$, Séminaire Bourbaki 44-ème année (1991-92), 759.

[9] Scherk (H. F.) J. R. Angew Math. 13 (1835), p. 185.

[10] SCHOEN (R.) .- Uniqueness, symmetry ans embeddedness of minimal surfaces, J. Diff. Geom 18 (1983), pp. 791-809.

[11] WEI (F.) . - Some existence and uniqueness theorems for doubly periodic minimal surfaces, Invent. Math 109 (1992), pp. 113-136. 